\title{
TOPOLOGICALLY STATIONARY LOCALLY COMPACT GROUPS AND AMENABILITY
}

\author{
BY \\ JAMES C. S. WONG( $\left.{ }^{1}\right)$
}

1. Introduction. A semigroup $S$ is called right stationary if for each $f \in m(S)$, the weak* closed convex hull of right translates of $f$ in $m(S)=l_{1}(S)^{*}$ contains a constant function. In [18], T. Mitchell proved that a semigroup $S$ is right stationary iff $m(S)$ has a left invariant mean. In this case, the set of values $\mu(f)$ where $\mu$ runs over all left invariant means on $m(S)$ coincides with the set of constants in the weak* closed convex hull of right translates of $f$. The main purpose of this paper is to prove a topological analogue (which is also a generalisation) of this theorem for locally compact groups. It turns out that our analogue of Mitchell's theorem is a consequence of a more general "Localisation Theorem" on invariant means which we are going to present in $§ 5$. An application of this "Localisation Theorem" and its consequences is given in $\S 6$ while the rest of the paper is devoted to proving a theorem on topological left almost convergence as well as some further generalisations of discrete theory.

2. Notations. For general terms in harmonic analysis, we shall follow Hewitt and Ross [14] (unless otherwise stated explicitly). Let $G$ be a locally compact group with a fixed left Haar measure $\lambda$ and modular function $\Delta$. Symbols like $\int \cdots d x$ or $\int \cdots d y$ will always denote integration with respect to $\lambda$. Let $B M(G)$ be the algebra (pointwise operations) of all real valued bounded $\lambda$-measurable functions on $G$ with supremum norm $\|\quad\|_{u}$ and $N$ the closed ideal of all locally null functions in $B M(G)\left(\left[14\right.\right.$, Definition 11.26]). Let $L_{\infty}(G)=B M(G) / N$ be the quotient Banach algebra with quotient norm \|\|$_{\infty}$ (i.e. essential supremum norm).

A linear functional $m$ in $L_{\infty}(G)^{*}$ is called a mean if ess inf $f \leqq m(f) \leqq \operatorname{ess} \sup f$ for any $f \in L_{\infty}(G)$ (see $\left.[12, \S 2.1]\right)$. A mean $m$ on $L_{\infty}(G)$ is topological left (right) invariant if for every $f \in L_{\infty}(G), \varphi \in P(G)=\left\{\varphi \in L_{1}(G): \varphi \geqq 0,\|\varphi\|_{1}=1\right\}$, we have $m(\varphi * f)=m(f)\left(m\left(f * \varphi^{\sim}\right)=m(f)\right)$. Here $f^{\sim}$ is the function defined by $f^{\sim}(x)$ $=f\left(x^{-1}\right), x \in G\left({ }^{2}\right) . m$ is topological invariant if it is both topological left and topological right invariant. If $X$ is a linear subspace of $L_{\infty}(G)$ containing the constants, $X$ is said to be topological left (right) invariant if

$$
P(G) * X \subset X \quad(X * P(G) \sim X) .
$$

Received by the editors January 15, 1969 and, in revised form, April 9, 1969.

( ${ }^{1}$ This research was supported in part by a postgraduate fellowship of the National Research Council of Canada. It contains parts of the results from the author's doctoral thesis at the University of British Columbia written under the direction of Professor E. E. Granirer.

${ }^{2}$ ) Note that in Hewitt and Ross [14], the notation $f^{*}$ is used instead of $f^{\sim}$. The latter is taken from Greenleaf [12] and Hulanicki [15]. 
Topological left invariant means (TLIM) and topological right invariant means (TRIM) on such linear subspaces $X$ of $L_{\infty}(G)$ can be defined similarly.

For $f \in L_{\infty}(G), g \in L_{1}(G)$, let $(f, g)$ denote the defining bilinear functional of the pair $L_{\infty}(G)$ and $L_{1}(G)$. If $g \in L_{1}(G), s \in G$, the maps $l_{s}, r_{s}, l_{g}, r_{g}: L_{\infty}(G) \rightarrow L_{\infty}(G)$ are defined by $l_{s} f(t)=f(s t), r_{s} f(t)=f(t s), l_{g}(f)=(1 / \Delta) g^{\sim} * f$ and $r_{g}(f)=f * g^{\sim}$ for any $f \in L_{\infty}(G), t \in G$. ( $\left(l_{s} f\right.$ is sometimes written as ${ }_{s} f$ and $r_{s} f$ as $f_{s}$.) It is clear that the maps $l_{s}, r_{s}, l_{g}$ and $r_{g}$ are (norm) continuous and linear and satisfy $\left\|l_{s}\right\|=\left\|r_{s}\right\|=1$, $\left\|l_{g}\right\|,\left\|r_{g}\right\| \leqq\|g\|_{1}$.

Let $C B(G)$ be the Banach space of all real valued bounded continuous functions on $G$ and let $U C B_{r}(G)\left(U C B_{l}(G)\right)$ consist of all $f \in C B(G)$ such that the map $s \rightarrow l_{s} f\left(s \rightarrow r_{s} f\right)$ from $G$ into $C B(G)$ is continuous when $C B(G)$ has the usual supremum norm. Functions in $U C B_{r}(G)\left(U C B_{l}(G)\right)$ are called right (left) uniformly continuous. Notice that some authors call functions in $U C B_{r}(G)\left(U C B_{l}(G)\right)$ left (right) uniformly continuous and denote them by $L U C(G)(R U C(G))$, see for example [9] and [21].

3. Technical lemmas. In this section, we state a number of lemmas which are needed in proving the main theorems. Most of these are quite well-known. For completeness we shall bring in the proofs.

LEMMA 3.1. Let $G$ be a locally compact group, then

(a) The mapping $g \rightarrow(1 / \Delta) g^{\sim}$ is a linear isometry of $L_{1}(G)$ onto itself. It is also a homeomorphism when $L_{1}(G)$ has the weak topology.

(b) The mapping $f \rightarrow f^{\sim}$ is a linear isometry of $L_{\infty}(G)$ onto itself. It is also a homeomorphism when $L_{\infty}(G)$ has the weak* topology.

(c) For any $f \in L_{\infty}(G), g, \varphi \in L_{1}(G)$, we have

$$
\left(f * g^{\sim}, \varphi\right)=(f, \varphi * g)=\left((1 / \Delta) \varphi^{\sim} * f, g\right) .
$$

(d) For any $g, \varphi \in L_{1}(G),(1 / \Delta)(\varphi * g)^{\sim}=(1 / \Delta) g^{\sim} *(1 / \Delta) \varphi^{\sim}$.

(e) For any $f \in L_{\infty}(G), \varphi \in L_{1}(G), f^{\sim} * \varphi^{\sim}=(\varphi * f)^{\sim}$.

Proof. (a) By [14, Theorem 20.2], we see that $g \rightarrow(1 / \Delta) g^{\sim}$ is a linear isometry of $L_{1}(G)$ onto itself. Since it is self-inversed, the second part of (a) is immediate.

(b) Let $T: L_{1}(G) \rightarrow L_{1}(G)$ be the map in (a). Consider its adjoint $T^{*}: L_{\infty}(G) \rightarrow$ $L_{\infty}(G)$. If $f \in L_{\infty}(G)$ and $g \in L_{1}(G)$, then $\left(T^{*} f\right) g=\left(f,(1 / \Delta) g^{\sim}\right)=\left(f^{\sim}, g\right)$ by [14, Theorem 20.2]. Hence $T^{*} f=f^{\sim}$. Since inversion in $G$ preserves locally null sets, $\left\|f^{\sim}\right\|_{\infty}=\|f\|_{\infty}$ and the map $f \rightarrow f^{\sim}$ is a linear isometry which is self-inversed. Hence being the adjoint of $T$, it is also a homeomorphism when $L_{\infty}(G)$ has the weak* topology.

(c) Let $f \in L_{\infty}(G), g, \varphi \in L_{1}(G)$, we have

$$
\begin{aligned}
\left(f * g^{\sim}, \varphi\right) & =\int\left(f * g^{\sim}\right)(x) \varphi(x) d x=\iint f(y) g^{\sim}\left(y^{-1} x\right) d y \varphi(x) d x \\
& =\iint g\left(x^{-1} y\right) \varphi(x) d x f(y) d y=\int(\varphi * g)(y) f(y) d y=(f, \varphi * g),
\end{aligned}
$$


by [14, Corollary 20.14(iii)], Fubini's Theorem and [14, Theorem 20.10(i)] respectively. On the other hand

$$
\begin{aligned}
\left((1 / \Delta) \varphi^{\sim} * f, g\right) & =\int\left((1 / \Delta) \varphi^{\sim} * f\right)(x) g(x) d x=\iint \Delta\left(y^{-1}\right) \varphi\left(y^{-1}\right) f\left(y^{-1} x\right) d y g(x) d x \\
& =\iint \varphi(y) f(y x) d y g(x) d x=\iint f(y x) g(x) d x \varphi(y) d y \\
& =\int\left(f * g^{\sim}\right)(y) \varphi(y) d y=\left(f * g^{\sim}, \varphi\right)
\end{aligned}
$$

by [14, Corollary 20.14(i), Theorem 20.2], Fubini's Theorem and [14, Corollary 20.14(iii)] respectively in the second through fifth equalities.

(d) The map $g \rightarrow(1 / \Delta) g^{\sim}$ is an involution of the group algebra $L_{1}(G)$ (with convolution as multiplication) as is well-known.

(e) Let $f \in L_{\infty}(G), \varphi \in L_{1}(G)$, then

$$
\begin{aligned}
\left(f^{\sim} * \varphi^{\sim}\right)(x) & =\int f^{\sim}(y) \varphi^{\sim}\left(y^{-1} x\right) d y=\int f\left(y^{-1}\right) \varphi\left(x^{-1} y\right) d y \\
& =\int f\left(y^{-1} x^{-1}\right) \varphi(y) d y=(\varphi * f)^{\sim}(x) .
\end{aligned}
$$

LEMMA 3.2. Let $G$ be a locally compact group, then

(a) For fixed $\varphi \in L_{1}(G)$, the map $f \rightarrow \varphi * f$ of $L_{\infty}(G)$ into itself is $w^{*}-w^{*}$ continuous.

(b) For fixed $f \in L_{\infty}(G)$, the map $\varphi \rightarrow \varphi *$ fof $L_{1}(G)$ into $L_{\infty}(G)$ is $w-w^{*}$ continuous.

Similar assertions hold for the mappings $f \rightarrow f * \varphi^{\sim}$ and $\varphi \rightarrow f * \varphi^{\sim}$.

Proof. (a) Let $\varphi \in L_{1}(G)$ be fixed. Define a map $T: L_{1}(G) \rightarrow L_{1}(G)$ by $T g=$ $(1 / \Delta) \varphi^{\sim} * g, g \in L_{1}(G)$. Clearly $T$ is a bounded linear mapping. Consider its adjoint $T^{*}: L_{\infty}(G) \rightarrow L_{\infty}(G)$. We have $\left(T^{*} f\right) g=\left(f,(1 / \Delta) \varphi^{\sim} * g\right)=(\varphi * f, g)$ by Lemma 3.1(c). Hence $T^{*} f=\varphi * f$. Thus the map $f \rightarrow \varphi * f$ being the adjoint of $T$ is $w^{*}-w^{*}$ continuous.

(b) Let $X, Y$ be Banach spaces and $T: X \rightarrow Y^{*}$ be bounded linear. Then $T: X \rightarrow Y^{*}$ is $w$-w continuous by [6, Theorem 15, p. 422] and a fortiori $w-w^{*}$ continuous. Take $X=L_{1}(G), Y=L_{\infty}(G)$ and $T \varphi=\varphi * f$, the result follows.

The assertions about the maps $f \rightarrow f * \varphi^{\sim}$ and $\varphi \rightarrow f * \varphi^{\sim}$ can be proved similarly.

LemmA 3.3. Let $\left\{\varphi_{\alpha}\right\}$ be an approximate identity in $L_{1}(G)$ (see $[14$, Definition 20.26]), then

(a) $\left\{(1 / \Delta) \varphi_{\alpha}^{\tilde{\alpha}}\right\}$ is also an approximate identity in $L_{1}(G)$.

(b) If $f \in L_{\infty}(G)$, then $w^{*} \lim _{\alpha} \varphi_{\alpha} * f=f=w^{*} \lim _{\alpha} f * \varphi_{\alpha}^{\tilde{\alpha}}$.

Proof. (a) If $\varphi \in L_{1}(G), \psi=(1 / \Delta) \varphi^{\sim} \in L_{1}(G)$. Hence

$$
(1 / \Delta) \varphi_{\alpha}^{\tilde{\alpha}} * \varphi=(1 / \Delta) \varphi_{\alpha}^{\tilde{\alpha}} *(1 / \Delta) \psi^{\sim}=(1 / \Delta)\left(\psi * \varphi_{\alpha}\right)^{\sim} \rightarrow(1 / \Delta) \psi^{\sim}=\varphi
$$


in norm topology of $L_{1}(G)$ by Lemma 3.1(d) and (a). Similarly $\varphi *(1 / \Delta) \varphi_{\alpha} \tilde{\alpha} \rightarrow \varphi$ in norm topology of $L_{1}(G)$.

(b) Let $\left\{\varphi_{\alpha}\right\}$ be an approximate identity in $L_{1}(G)$. By (a) so is $\left\{(1 / \Delta) \varphi_{\alpha}^{\tilde{\alpha}}\right\}$. If $f \in L_{\infty}(G)$, then for any $g \in L_{1}(G),(1 / \Delta) \varphi_{\alpha} * g \rightarrow g$ in norm topology of $L_{1}(G)$. In particular $(1 / \Delta) \varphi_{\alpha}^{\tilde{\alpha}} * g \rightarrow g$ weakly in $L_{1}(G)$. Hence $\left(\varphi_{\alpha} * f, g\right)=\left(f,(1 / \Delta) \varphi_{\alpha} * g\right) \rightarrow$ $(f, g)$ by Lemma 3.1(c). Consequently $\varphi_{\alpha} * f \rightarrow f$ in weak* topology of $L_{\infty}(G)$. Similarly $f * \varphi_{\alpha} \rightarrow f$ in weak ${ }^{*}$ topology of $L_{\infty}(G)$.

4. Arens product. In [1], R. Arens has shown how to define an associative multiplication in the second conjugate space $B^{* *}$ of a Banach algebra $B$. This multiplication renders $B^{* *}$ a Banach algebra and extends the multiplication in $B$. We shall describe the procedure in the special case when $B$ is the group algebra $L_{1}(G)$ with convolution as multiplication. This Arens product plays an important role in subsequent discussions. See also Civin and Yood [25].

For $f \in L_{\infty}(G), g \in L_{1}(G)$ we define $f \odot g \in L_{\infty}(G)$ by $(f \odot g) \varphi=(f, g * \varphi)$ for each $\varphi \in L_{1}(G)$.

For $\nu \in L_{\infty}(G)^{*}, f \in L_{\infty}(G)$, we define $\nu \odot f \in L_{\infty}(G)$ by $(\nu \odot f) g=\nu(f \odot g)$ for each $g \in L_{1}(G)$.

For $\mu, \nu \in L_{\infty}(G)^{*}$, we define $\mu \odot \nu \in L_{\infty}(G)^{*}$ by $(\mu \odot \nu) f=\mu(\nu \odot f)$ for each $f \in L_{\infty}(G)$.

The following lemma consists of a multitude of results analogous to those in M. M. Day's classical paper on amenable semigroups $[2, \S 6]$. The proofs are also "topological variants" of those in the same paper.

LEMMA 4.1. (a) Let $\nu \in L_{\infty}(G)^{*}, f \in L_{\infty}(G), \varphi, g \in L_{1}(G)$. Then $f \odot g=(1 / \Delta) g^{\sim} * f$, $\varphi \odot f=f * \varphi^{\sim}$ and $\varphi \odot g=\varphi * g$.

(b) If $\mu, \nu$ are means on $L_{\infty}(G)$, so is $\mu \odot \nu$.

(c) If $\varphi \in L_{1}(G), \mu, \nu \in L_{\infty}(G)^{*}$. Then $\varphi \odot \nu=l_{\Phi}^{*} \nu, \mu \odot \varphi=r_{\phi}^{*} \mu$.

(d) For fixed $\varphi \in L_{1}(G)$, the maps $\mu \rightarrow \mu \odot \varphi$ and $\nu \rightarrow \varphi \odot \nu$ are $w^{*}-w^{*}$ continuous.

(e) For fixed $\nu \in L_{\infty}(G)^{*}$, the map $\mu \rightarrow \mu \odot \nu$ is $w^{*}-w^{*}$ continuous.

(f) $\mu \odot \nu$ is topological left invariant if $\mu$ is topological left invariant. It is topological right invariant if $\nu$ is topological right invariant.

(g) If $\nu$ is topological left invariant and $\mu$ is a mean on $L_{\infty}(G)$, then $\mu \odot \nu=\nu$.

Proof. (a) For any $\psi \in L_{\uparrow}(G),(f \odot g) \psi=(f, g * \psi)=\left((1 / \Delta) g^{\sim} * f, \psi\right)$ by Lemma 3.1(c). Hence $f \odot g=(1 / \Delta) g^{\sim} * f$. Also $(\varphi \odot f) \psi=\varphi(f \odot \psi)=\left((1 / \Delta) \psi^{\sim} * f, \varphi\right)$ $=\left(f * \varphi^{\sim}, \psi\right)$ by Lemma 3.1(c) again. Hence $\varphi \odot f=f * \varphi^{\sim}$. Finally if $f \in L_{\infty}(G)$, $(\varphi \odot g) f=\varphi(g \odot f)=\varphi\left(f * g^{\sim}\right)=\left(f * g^{\sim}, \varphi\right)=(f, \varphi * g)=(\varphi * g) f$. Hence

$$
\varphi \odot g=\varphi * g .
$$

(b) Let $\mu, \nu$ be means on $L_{\infty}(G)$. Then clearly $\|\mu \odot \nu\| \leqq\|\mu\|\|\nu\|=1$. Now if 1 denotes the constant one function on $G$, we have $1 \odot g=(1 / \Delta) g^{\sim} * 1=\|g\|_{1} \cdot 1$ and $(\nu \odot 1) g=\nu(1 \odot g)=\|g\|_{1}=(1, g)$ for any $g \in L_{1}(G)$ and $g \geqq 0$. Hence by 
linearity $\nu \odot 1=1$ and therefore $(\mu \odot \nu) 1=\mu(\nu \odot 1)=\mu(1)=1$. Consequently $\mu \odot \nu$ is a mean on $L_{\infty}(G)$.

(c) We have $(\varphi \odot \nu) f=\varphi(\nu \odot f)=(\nu \odot f) \varphi=v(f \odot \varphi)=\nu\left((1 / \Delta) \varphi^{\sim} * f\right)=\nu\left(l_{\varphi} f\right)=$ $\left(l_{\omega}^{*} \nu\right) f$ for any $f \in L_{\infty}(G)$. Hence $\varphi \odot \nu=l_{\omega}^{*} \nu$. Also $(\mu \odot \varphi) f=\mu(\varphi \odot f)=\mu\left(f * \varphi^{\sim}\right)$ $=\mu\left(r_{\varphi} f\right)=\left(r_{\varphi}^{*} \mu\right) f$ for any $f \in L_{\infty}(G)$. So $\mu \odot \varphi=r_{\varphi}^{*} \mu$.

(d) This is immediate from (c).

(e) Suppose $\mu_{\alpha} \rightarrow \mu$ in weak* topology of $L_{\infty}(G)^{*}$. Then for any $f \in L_{\infty}(G)$, $\left(\mu_{\alpha} \odot \nu\right) f=\mu_{\alpha}(\nu \odot f) \rightarrow \mu(\nu \odot f)=(\mu \odot \nu) f$.

(f) Let $\mu$ be topological left invariant, then for any $\varphi \in P(G)$,

$$
l_{\Phi}^{*}(\mu \odot \nu)=\varphi \odot(\mu \odot \nu)=(\varphi \odot \mu) \odot \nu=\left(l_{\omega_{\alpha}}^{*} \mu\right) \odot \nu=\mu \odot \nu
$$

by (c) and by topological left invariance of $\mu$. If $\nu$ is topological right invariant, then for any $\varphi \in P(G), r_{\phi}^{*}(\mu \odot \nu)=(\mu \odot \nu) \odot \varphi=\mu \odot(\nu \odot \mu)=\mu \odot\left(r_{\phi}^{*} \nu\right)=\mu \odot \nu$ by similar arguments.

(g) Let $\mu$ be a mean on $L_{\infty}(G)$. By weak* density of the set $P(G)$ in the set of all means on $L_{\infty}(G)$ (see [15]), there is a net $\varphi_{\alpha}$ in $P(G)$ such that $\varphi_{\alpha} \rightarrow \mu$ in weak* topology of $L_{\infty}(G)^{*}$. Then $\varphi_{\alpha} \odot \nu \rightarrow \mu \odot \nu$ weak* by (e). Now $\varphi_{\alpha} \odot \nu=l_{\omega_{\alpha}}^{*} \nu=\nu$ by (c) and topological left invariance of $\nu$. Hence $\mu \odot \nu=\nu$.

Remark 4.1.1. Lemma 4.1(d) and (e) are due to Arens [1] for the general case. The assertion that $\varphi \odot g=\varphi * g$ is also proved in Arens [24] in the general case.

Definition 4.2. For each $m \in L_{\infty}(G)^{*}$, define a mapping $m_{L}: L_{\infty}(G) \rightarrow L_{\infty}(G)$ by $m_{L}(f) g=m\left((1 / \Delta) g^{\sim} * f\right)$ for any $f \in L_{\infty}(G), g \in L_{1}(G)$. The operator $m_{L}$ is called the topological left introversion of $m$. Similarly the topological right introversion $m_{R}: L_{\infty}(G) \rightarrow L_{\infty}(G)$ is defined by $m_{R}(f) g=m\left(f * g^{\sim}\right)$ for any $f \in L_{\infty}(G), g \in L_{1}(G)$.

LEMMA 4.3. (a) $m_{L}: L_{\infty}(G) \rightarrow L_{\infty}(G)$ is bounded linear, $\left\|m_{L}(f)\right\|_{\infty} \leqq\|m\|\|f\|_{\infty}$ and $m_{L}(f)=m \odot f$ for any $f \in L_{\infty}(G)$.

(b) If $\nu \in L_{\infty}(G)^{*}, f \in L_{\infty}(G), \varphi, g \in L_{1}(G)$. Then $\varphi_{L}=r_{\varphi}, \nu_{L}(g * f)=g * \nu_{L}(f)$.

(c) If $m_{\alpha} \rightarrow m$ in norm topology of $L_{\infty}(G)^{*}$ then $\left(m_{\alpha}\right)_{L} \rightarrow m_{L}$ in uniform operator topology.

(d) If $m_{\alpha} \rightarrow m$ in weak* topology of $L_{\infty}(G)^{*}$, then for any $f \in L_{\infty}(G),\left(m_{\alpha}\right)_{L} f \rightarrow$ $m_{L}(f)$ in weak* topology of $L_{\infty}(G)$.

Proof. (a) $\left|m_{L}(f) g\right|=\left|m\left((1 / \Delta) g^{\sim} * f\right)\right| \leqq\|m\|\|g\|_{1}\|f\|_{\infty}$. Hence

$$
\left\|m_{L}(f)\right\|_{\infty} \leqq\|m\|\|f\|_{\infty} \text {. }
$$

But $m_{L}(f) g=m\left((1 / \Delta) g^{\sim} * f\right)=m(f \odot g)=(m \odot f) g$ by Lemma 4.1(a). Thus $m_{L}(f)=m \odot f$.

(b) If $\varphi \in L_{1}(G), \varphi_{L}(f)=\varphi \odot f=r_{\varphi} f$ by (a) and Lemma 4.1(a). Hence $\varphi_{L}=r_{\varphi}$. If $f \in L_{\infty}(G), g \in L_{1}(G)$ and $\nu \in L_{\infty}(G)^{*}$, then

$$
\begin{aligned}
\nu_{L}(g * f) \varphi & =\nu\left(\frac{1}{\Delta}\left(\frac{1}{\Delta} g^{\sim} * \varphi\right)^{\sim} * f\right)=\nu_{L}(f)\left(\frac{1}{\Delta} g^{\sim} * \varphi\right)=\left(\nu_{L}(f), \frac{1}{\Delta} g^{\sim} * \varphi\right) \\
& =\left(g * \nu_{L}(f), \varphi\right)
\end{aligned}
$$

for any $\varphi \in L_{1}(G)$, by Lemma 3.1(a), (c) and (d). Hence $\nu_{L}(g * f)=g * \nu_{L}(f)$. 
(c) Assume $m_{\alpha} \rightarrow m$ in norm topology of $L_{\infty}(G)^{*}$. Since $\left(m_{\alpha}\right)_{L}-m_{L}=\left(m_{\alpha}-m\right)_{L}$, $\left\|\left(\left(m_{\alpha}\right)_{L}-m_{L}\right) f\right\| \leqq\left\|m_{\alpha}-m\right\|\|f\|_{\infty}$ by (a). Hence $\left\|\left(m_{\alpha}\right)_{L}-m_{L}\right\| \leqq\left\|m_{\alpha}-m\right\| \rightarrow 0$.

(d) Let $m_{\alpha} \rightarrow m$ in weak* topology of $L_{\infty}(G)^{*}$. Then for any $f \in L_{\infty}(G), g \in L_{1}(G)$, $\left(\left(m_{\alpha}\right)_{L} f\right) g-\left(m_{L} f\right) g=m_{\alpha}\left((1 / \Delta) g^{\sim} * f\right)-m\left((1 / \Delta) g^{\sim} * f\right) \rightarrow 0$.

REMARK. Lemma 4.3 has an analogue for topological right introversion (with some modifications). In fact we have $\varphi_{R}=l_{\varphi}, \nu_{R}\left(f * g^{\sim}\right)=\nu_{R}(f) * g^{\sim}$ and $m_{R} f=$ $m \odot_{R} f$ where $\bigcirc_{R}$ is the Arens product arising from the Banach algebra $L_{1}(G)$ where multiplication is convolution with the order of factors interchanged. The rest of Lemma 4.3 remains valid if we replace $L$ by $R$. We shall not need these results in what follows.

\section{Main theorems.}

Definition 5.1. A linear subspace $X$ of $L_{\infty}(G)$ is said to be topological left introverted if for any mean $m$ on $L_{\infty}(G), m_{L}(X) \subset X$. (Thus by definition of $m_{L}$, $L_{\infty}(G)$ is always topological left introverted.)

TheOREM 5.2 (Localisation). Let $X$ be a topological left introverted and topological left invariant linear subspace of $L_{\infty}(G)$ containing the constants. Then $X$ has a topological left invariant mean iff for any $f \in X$, there is a mean $m_{f}$ on $X$ such that $m_{f}(\varphi * f)=m_{f}(f)$ for every $\varphi \in P(G)$.

Proof. Necessity is trivial. To prove sufficiency, define for each $f \in X, K_{f}=\{m: m$ is a mean on $L_{\infty}(G), m(\varphi * f)=m(f)$ for any $\left.\varphi \in P(G)\right\}$. By assumption $K_{f} \neq \varnothing$ (since any mean on $X$ can be extended to a mean on $L_{\infty}(G)$ by Hahn Banach Theorem). Clearly $K_{f}$ is a weak* closed subset of the norm closed unit ball in $L_{\infty}(G)^{*}$ which is weak* compact. We show that the family $\left\{K_{f}: f \in X\right\}$ has the finite intersection property. Let $f_{1}, f_{2}, \ldots, f_{n} \in X$. When $n=1$, this is clear. Assume $\bigcap_{i=1}^{n-1} K_{f_{i}} \neq \varnothing$ and let $m \in \bigcap_{i=1}^{n-1} K_{f_{i}}$. Define $f=m_{L}\left(f_{n}\right) \in X$ since $X$ is topological left introverted. Let $\mu \in K_{f}$ and consider the mean $\mu \odot m$ (Lemma 4.1(b)). We claim that $\mu \odot m \in \bigcap_{i=1}^{n} K_{f_{i}}$.

For $1 \leqq i \leqq n-1$, if $\varphi \in P(G), m_{L}\left(f_{i}\right) \varphi=m\left((1 / \Delta) \varphi^{\sim} * f_{i}\right)=m\left(f_{i}\right)$ since $m \in K_{f_{i}}$. Therefore $\left(m_{L}\left(f_{i}\right), \varphi\right)=\left(m\left(f_{i}\right) \cdot 1, \varphi\right)$ for any $\varphi \in P(G)$ which spans $L_{1}(G)$. Hence $m_{L}\left(f_{i}\right)=m\left(f_{i}\right) \cdot 1$ and it follows that if $\varphi \in P(G)$,

$$
\begin{aligned}
(\mu \odot m)\left(\varphi * f_{i}\right) & =\mu\left(m \odot\left(\varphi * f_{i}\right)\right)=\mu\left(m_{L}\left(\varphi * f_{i}\right)\right)=\mu\left(\varphi * m_{L}\left(f_{i}\right)\right) \\
& =\mu\left(\varphi * m\left(f_{i}\right) \cdot 1\right)=\mu\left(m\left(f_{i}\right) \cdot 1\right)=\mu\left(m_{L}\left(f_{i}\right)\right)=(\mu \odot m) f_{i},
\end{aligned}
$$

where we have made use of Lemma 4.3(a) and (b) respectively in the second and third equality. On the other hand, if $\varphi \in P(G)$,

$$
\begin{aligned}
(\mu \odot m)\left(\varphi * f_{n}\right) & =\mu\left(m_{L}\left(\varphi * f_{n}\right)\right)=\mu\left(\varphi * m_{L}\left(f_{n}\right)\right)=\mu(\varphi * f)=\mu(f) \quad\left(\text { since } \mu \in K_{f}\right) \\
& =\mu\left(m_{L}\left(f_{n}\right)\right)=(\mu \odot m) f_{n} .
\end{aligned}
$$

Consequently $\mu \odot m \in \bigcap_{i=1}^{n} K_{f_{i}}$. By weak* compactness of the norm closed unit ball in $L_{\infty}(G)^{*}, \bigcap\left\{K_{f}: f \in X\right\} \neq \varnothing$. Any mean in this intersection is necessarily a topological left invariant mean on $X$. 
Remark. For discrete groups $G$, this theorem is due to E. Granirer and Anthony Lau [11] and the above proof follows the idea in [11]. The corresponding theorem there is true for semigroups. There is also another version of the Localisation Theorem which is formally stronger. In fact, $X$ has a topological left invariant mean iff for each $f \in X$ and $\varphi \in P(G)$, there is a mean (depending on $f$ and $\varphi$ ) on $X$ such that $m(\psi *(f-\varphi * f))=0$ for any $\psi \in P(G)$. The proof is almost the same. We consider the sets $K(f, \varphi)=\left\{m: m\right.$ is a mean on $L_{\infty}(G)$ and $m(\psi *(f-\varphi * f))=0$ for any $\psi \in P(G)\}$ instead of $K_{f}$, use compactness to find a mean

$$
m \in \bigcap\{K(f, \varphi): f \in X, \varphi \in P(G)\} \text {. }
$$

Then $m$ satisfies the condition $m_{L}(\varphi * f)=m_{L}(f)$ for any $\varphi \in P(G), f \in X$, and $\mu=m \odot m$ is a topological left invariant mean on $X$. For discrete groups, this stronger version can also be found in [11].

Definition 5.3. Let $X$ be a linear subspace of $L_{\infty}(G)$, we say that $X$ is topologically right stationary iff for each $f \in X$, there is a net $\varphi_{\alpha} \in P(G)$ such that $f * \varphi_{\alpha}^{\tilde{\alpha}}$ converges weak* to a constant function in $L_{\infty}(G) . G$ is called topologically right stationary iff $L_{\infty}(G)$ is topologically right stationary in analogy to the definitions in Mitchell [18, §3].

For any $f \in L_{\infty}(G)$, define in analogy to Mitchell [18], $Z_{R}(f)$ as the weak* closure of $\left\{r_{\varphi} f: \varphi \in P(G)\right\}$ (or $w^{*} \mathrm{CL}\left(f * P(G)^{\sim}\right)$ ) and $K_{R}(f)=\left\{\alpha: \alpha\right.$ real, $\left.\alpha \cdot 1 \in Z_{R}(f)\right\}$. The set $K_{R}(f)$ may be empty. In these notations, $X$ is topologically right stationary iff $K_{R}(f) \neq \varnothing$ for any $f \in X$.

THEOREM 5.4. Let $X$ be a topological left introverted and topological left invariant linear subspace of $L_{\infty}(G)$ containing the constants. Then $X$ has a topological left invariant mean iff $X$ is topologically right stationary. In this case, for any $f \in X$, $\beta \in K_{R}(f)$ iff there is a topological left invariant mean $\mu$ on $X$ such that $\mu(f)=\beta$.

Proof. Assume that $X$ is topological right stationary, $f \in X$. Then there is a net $\varphi_{\alpha}$ in $P(G)$ such that $f * \varphi_{\alpha}^{\tilde{\alpha}}$ converges weak* to a constant function $\beta \cdot 1$ in $L_{\infty}(G)$. Passing to a subnet if necessary, we can assume that $\varphi_{\alpha}$ converges weak* to some mean $m$ in $L_{\infty}(G)^{*}$ by weak* compactness of the set of means in $L_{\infty}(G)^{*}$. Consider the mean $m \odot m$ on $L_{\infty}(G)$. We show that $m \odot m \in K_{f}$ (defined as in the proof of Theorem 5.2). Observe that for any $g \in L_{1}(G)$,

$$
\begin{aligned}
m_{L}(f) g & =m\left((1 / \Delta) g^{\sim} * f\right)=\lim _{\alpha} \varphi_{\alpha}\left((1 / \Delta) g^{\sim} * f\right)=\lim _{\alpha}\left((1 / \Delta) g^{\sim} * f, \varphi_{\alpha}\right) \\
& =\lim _{\alpha}\left(f * \varphi_{\alpha}^{\tilde{\alpha}}, g\right)=(\beta \cdot 1, g)
\end{aligned}
$$

by Lemma 3.1(c). Hence $m_{L}(f)=\beta \cdot 1$. Now

$$
\begin{aligned}
(m \odot m)(\varphi * f) & =m\left(m_{L}(\varphi * f)\right)=m\left(\varphi * m_{L}(f)\right)=m(\varphi * \beta \cdot 1)=m(\beta \cdot 1) \\
& =m\left(m_{L}(f)\right)=(m \odot m) f
\end{aligned}
$$

for any $\varphi \in P(G)$. Consequently $m \odot m \in K_{f}$. By Theorem 5.2, $X$ has a topological left invariant mean $\nu \in \bigcap\left\{K_{f}: f \in X\right\}$. Let $\mu=\nu \odot m$, then $\mu(f)=(\nu \odot m) f=$ 
$\nu\left(m_{L}(f)\right)=\nu(\beta \cdot 1)=\beta$. Since $\nu$ is a topological left invariant mean, so is $\mu$. For if $f \in X, \varphi \in P(G)$,

$$
\begin{aligned}
\mu(\varphi * f) & =(\nu \odot m)(\varphi * f)=\nu\left(m_{L}(\varphi * f)\right)=\nu\left(\varphi * m_{L}(f)\right)=\nu\left(m_{L}(f)\right) \\
& =(\nu \odot m) f=\mu(f),
\end{aligned}
$$

since $m_{L}(f) \in X$. (Note that we cannot invoke Lemma 4.1(f) directly because the mean $\nu$ is topological left invariant on $X$ only, but not necessarily on $L_{\infty}(G)$.)

Conversely, assume that $X$ has a topological left invariant mean $\mu$. Then there is a net $\varphi_{\alpha}$ in $P(G)$ such that $\varphi_{\alpha} \rightarrow \mu$ in weak* topology of $X^{*}$. Let $f \in X$, then

$$
\begin{aligned}
\left(f * \varphi_{\alpha}^{\tilde{\alpha}}, \varphi\right) & =\left((1 / \Delta) \varphi^{\sim} * f, \varphi_{\alpha}\right)=\varphi_{\alpha}\left((1 / \Delta) \varphi^{\sim} * f\right) \rightarrow \mu\left((1 / \Delta) \varphi^{\sim} * f\right)=\mu(f) \\
& =(\mu(f) \cdot 1, \varphi)
\end{aligned}
$$

for any $\varphi \in P(G)$ which spans $L_{1}(G)$. Here we have made use of Lemma 3.1(c) and topological left invariance of $\mu$. Hence $f * \varphi_{\alpha}^{\tilde{\alpha}}$ converges weak* to the constant function $\mu(f) \cdot 1$ in $L_{\infty}(G)$. In fact $\mu(f) \in K_{R}(f)$.

REMARKS. (a) If $X$ is a linear subspace of $L_{\infty}(G)$, then $X$ is topologically left introverted implies that $X$ is topologically right invariant. This is clear since

$$
f * \varphi^{\sim}=r_{\varphi} f=\varphi_{L}(f) \text {. }
$$

(b) It is possible to prove Theorem 5.4 (at least for the case $X=L_{\infty}(G)$ ) by a method similar to that employed in [9] or in [18].

(c) For discrete groups $G$, it is readily seen that

$$
\begin{aligned}
\mathrm{w}^{*} \mathrm{CL}\left\{f * \varphi^{\sim}: \varphi \in P(G)\right\} & =\mathrm{w}^{*} \mathrm{CLCO}\left\{r_{a} f: a \in G\right\} \\
& =\text { pointwise CL CO }\left\{r_{a} f: a \in G\right\}
\end{aligned}
$$

(see [18, Lemma 3]). Thus Theorem 5.4 reduces to a result of Mitchell's [18, Theorem 3] for groups.

6. An application. We now indicate how we can obtain a result in [11] from Theorem 5.4 by specialising $X=U C B_{r}(G)$ (or $L U C(G)$ in the notation of [11]). We shall need the following lemmas which are of independent interest.

LEMMA 6.1. Let $\dot{m} \in L_{\infty}(G)^{*}, f \in L_{\infty}(G)$ and $s \in G$. Then $l_{s}\left(m_{L}(f)\right)=m_{L}\left(l_{s} f\right)$.

Proof. If $g \in L_{1}(G)$, we have $\left(l_{s}\left(m_{L}(f)\right), g\right)=\left(m_{L}(f), s_{s}^{-1} g\right)=m\left((1 / \Delta)\left(s_{s}-1 g\right)^{\sim} * f\right)$ $=m\left(\Delta(s)\left((1 / \Delta) g^{\sim}\right)_{s} * f\right)=m\left((1 / \Delta) g^{\sim} *_{s} f\right)=\left(m_{L}\left(l_{s} f\right), g\right)$ by [14, Remark 20.11]. Hence $l_{s}\left(m_{L}(f)\right)=m_{L}\left(l_{s} f\right)$.

LEMMA 6.2. $U C B_{r}(G)$ is topological left introverted.

Proof. Let $s_{\alpha} \rightarrow s$ in $G, f \in U C B_{r}(G)$. Then $\left\|l_{s_{\alpha}} f-l_{s} f\right\|_{\infty} \rightarrow 0$. Hence if $m$ is any mean on $L_{\infty}(G),\left\|l_{s_{\alpha}}\left(m_{L}(f)\right)-l_{s}\left(m_{L}(f)\right)\right\|_{\infty}=\left\|m_{L}\left(l_{s_{\alpha}} f\right)-m_{L}\left(l_{s} f\right)\right\|_{\infty} \leqq\left\|m_{L}\left(l_{s_{\alpha}} f-l_{s} f\right)\right\|_{\infty}$ $\leqq\left\|l_{s_{\alpha}} f-l_{s} f\right\|_{\infty} \rightarrow 0$ by Lemma 6.1 and Lemma 4.3(a). Hence $m_{L}(f) \in U C B_{r}(G)$.

LemMA 6.3. For any $f \in L_{\infty}(G), \mathrm{w}^{*} \mathrm{CL}\left(f * P(G)^{\sim}\right)=\mathrm{w}^{*} \mathrm{CL} \mathrm{CO}\left\{r_{a} f: a \in G\right\}$. 
Proof. Let $a \in G$ and let $\varphi_{\alpha}$ be an approximate identity in $L_{1}(G)$. If $f \in L_{\infty}(G)$, then $f * \varphi_{\alpha}^{\tilde{\alpha}} \rightarrow f$ weak ${ }^{*}$ in $L_{\infty}(G)$. By $w^{*}-w^{*}$ continuity of $r_{a}$, we have $\left(f * \varphi_{\alpha}\right)_{a} \rightarrow$ $f_{a}=r_{a} f$ weak $^{*}$ in $L_{\infty}(G)$. But $\left(f * \varphi_{\alpha}^{\tilde{\alpha}}\right)_{a}=f *\left(\varphi_{\alpha}^{\tilde{\alpha}}\right)_{a}=f *\left(a^{-1} \varphi_{\alpha}\right) \sim \in f * P(G) \sim$. Hence $r_{a} f \in \mathrm{w}^{*} \mathrm{CL}\left(f * P(G)^{\sim}\right)$ which is weak* closed and convex. Therefore

$$
\mathrm{w}^{*} \mathrm{CL} \mathrm{CO}\left\{r_{a} f: a \in G\right\} \subset \mathrm{w}^{*} \mathrm{CL}(f * P(G) \sim) \text {. }
$$

Conversely, let $\varphi \in P(G)$. Then there is a net of finite means $\varphi_{\alpha}=\sum_{i=1}^{n_{\alpha}} \beta_{i}^{\alpha} \delta_{a_{i}^{\alpha}}\left({ }^{3}\right)$ such that $\varphi_{\alpha} \rightarrow \varphi$ in weak* topology of $C B(G)^{*}$. If $g \in L_{1}(G)$, then

$$
\begin{aligned}
\left(f * \varphi^{\sim}, g\right)=\left(\frac{1}{\Delta} g^{\sim} * f, \varphi\right) & =\lim _{\alpha} \varphi_{\alpha}\left(\frac{1}{\Delta} g^{\sim} * f\right)=\lim _{\alpha} \sum_{i=1}^{n_{\alpha}} \beta_{i}^{\alpha}\left(\frac{1}{\Delta} g^{\sim} * f\right)\left(a_{i}^{\alpha}\right) \\
& =\lim _{\alpha} \sum_{i=1}^{n_{\alpha}} \beta_{i}^{\alpha} \int f\left(y a_{i}^{\alpha}\right) g(y) d y=\lim _{\alpha}\left(\sum_{i=1}^{n_{\alpha}} \beta_{i}^{\alpha} r_{a_{i}^{\alpha}} f, g\right) .
\end{aligned}
$$

(Here we have made use of Lemma 3.1(c), [14, Corollary 20.14 and Theorem 20.2].) Hence $f * \varphi^{\sim} \in \mathrm{w}^{*} \mathrm{CL} \operatorname{CO}\left\{r_{a} f: a \in G\right\}$ which is weak* closed and convex. Hence $\mathrm{w}^{*} \mathrm{CL}\left(f * P(G)^{\sim}\right) \subset \mathrm{w}^{*} \mathrm{CL} \mathrm{CO}\left\{r_{a} f: a \in G\right\}$.

Remark. The inclusion $\mathrm{w}^{*} \mathrm{CL}\left(f * P(G)^{\sim}\right) \subset \mathrm{w}^{*} \mathrm{CL} \mathrm{CO}\left\{r_{a} f: a \in G\right\}$ was suggested by Professor M. M. Day (written communication). The above proof is different from his. The author wishes to thank Professor Day for this important suggestion.

LEMMA 6.4. Let $\tau_{c}$ be the topology of uniform convergence on compacta. For each $f \in U C B_{r}(G), \mathrm{w}^{*} \mathrm{CL}\left(f * P(G)^{\sim}\right)=\tau_{c} \operatorname{CL~CO}\left\{r_{a} f: a \in G\right\}$.

Proof. We first observe that if $f \in U C B_{r}(G)$, then $\operatorname{CO}\left\{r_{a} f: a \in G\right\}$ is a bounded equicontinuous family of functions by a straightforward argument. The same is true for the family $F=\tau_{c} \operatorname{CL~CO}\left\{r_{a} f: a \in G\right\}$ by [16, Theorem 7.14]. Let $\varphi \in P(G)$. Then there is a net $\varphi_{\alpha}=\sum_{i=1}^{n_{\alpha}} \beta_{i}^{\alpha} \delta_{a_{i}^{\alpha}}$ of finite means such that $\varphi_{\alpha} \rightarrow \varphi$ in weak* topology of $C B(G)^{*}$. Now

$$
f * \varphi^{\sim}(x)=\int f(x y) \varphi(y) d y=\left({ }_{x} f, \varphi\right)=\lim _{\alpha} \varphi_{\alpha}\left({ }_{x} f\right)=\lim _{\alpha} \sum_{i=1}^{n_{\alpha}} \beta_{i}^{\alpha} r_{a_{i}^{\alpha}} f(x) .
$$

Hence there is a net $f_{\alpha}=\sum_{i=1}^{n_{\alpha}} \beta_{i}^{\alpha} r_{a_{i}^{\alpha}} f \in \mathrm{CO}\left\{r_{a} f ; a \in G\right\}$ such that $f_{\alpha} \rightarrow f * \varphi^{\sim}$ pointwise and hence in the $\tau_{c}$ topology by [16, Theorem 7.15]. Hence $f * P(G) \sim F$ which is $\tau_{c}$ compact by Ascoli's Theorem [16, Theorem 7.17]. Since the family $F$ is bounded in norm, the $\tau_{c}$ topology is finer than the weak* topology of $L_{\infty}(G)$ restricted to $F$. Since they are both Hausdorff, they must coincide on $F$. In particular $F$ is weak* closed in $L_{\infty}(G)$. Therefore $\mathrm{w}^{*} \mathrm{CL}\left(f * P(G)^{\sim}\right) \subset \tau_{c} \mathrm{CL} \mathrm{CO}\left\{r_{a} f: a \in G\right\}$.

On the other hand by Lemma $6.3 \mathrm{w}^{*} \mathrm{CL} \operatorname{CO}\left\{r_{a} f: a \in G\right\}=\mathrm{w}^{*} \mathrm{CL}\left(f * P(G)^{\sim}\right)$. But the family $\operatorname{CO}\left\{r_{a} f: a \in G\right\}$ is bounded. Hence

$$
\tau_{c} \operatorname{CLCO}\left\{r_{a} f: a \in G\right\} \subset \mathrm{w}^{*} \mathrm{CL} \operatorname{CO}\left\{r_{a} f: a \in G\right\}=\mathrm{w}^{*} \mathrm{CL}(f * P(G) \sim) .
$$

(3) If $a \in G, \delta_{a}$ is defined by $\delta_{a} f=f(a)$ for any $f \in C B(G)$. 
Consequently $\tau_{c} \operatorname{CLCO}\left\{r_{a} f: a \in G\right\}=\mathrm{w}^{*} \mathrm{CL}\left(f * P(G)^{\sim}\right)$.

TheOREM 6.5 (E. GRANIRER AND ANTHONY LAU [11]). UCB $(G)$ has a left invariant mean iff for each $f \in U C B_{r}(G), \tau_{c} \mathrm{CL} \mathrm{CO}\left\{r_{a} f: a \in G\right\}$ contains a constant function. In this case $\beta \cdot 1 \in \tau_{c} \operatorname{CL~CO}\left\{r_{a} f: a \in G\right\}$ iff there is a left invariant mean $\mu$ on $U C B_{r}(G)$ such that $\mu(f)=\beta$.

Proof. It was proved in [12] that TLIM and LIM on $U C B_{r}(G)$ are the same. By Lemma $6.4 \tau_{c} \mathrm{CL} \mathrm{CO}\left\{r_{a} f: a \in G\right\}=\mathrm{w}^{*} \mathrm{CL}(f * P(G) \sim)$. Theorem 6.5 is just Theorem 5.4 with $X=U C B_{r}(G)$.

7. Topological left almost convergence. In this section, we shall prove a theorem on topological left almost convergence. It is an analogue of a result in [10] on left almost convergence of bounded functions on semigroups. Let $G$ be a locally compact group, let $K$ denote the linear subspace of all functions in $L_{\infty}(G)$ which can be represented as $\sum_{i=1}^{n}\left(f_{i}-\varphi_{i} * f_{i}\right)$ where $f_{i} \in L_{\infty}(G), \varphi_{i} \in P(G), 1 \leqq i \leqq n$.

LEMMA 7.1. Let $G$ be a locally compact group, $m_{\alpha}$ a net of means on $L_{\infty}(G)$ such that $\lim _{\alpha}\left\|l_{\omega}^{*} m_{\alpha}-m_{\alpha}\right\|=0$ for any $\varphi \in P(G)$. Then for any $f \in K^{-}$(closure taken in norm topology of $\left.L_{\infty}(G)\right), \lim _{\alpha}\left\|\left(m_{\alpha}\right)_{R} f\right\|_{\infty}=0$ where $\left(m_{\alpha}\right)_{R}$ is the topological right introversion of $m_{\alpha}$.

Proof. Consider first functions in $K$ of the form $f-\varphi * f, f \in L_{\infty}(G), \varphi \in P(G)$. We have, if $g \in L_{1}(G)$,

$$
\begin{aligned}
\left|\left(m_{\alpha}\right)_{R}(f-\varphi * f) g\right| & =\left|m_{\alpha}\left((f-\varphi * f) * g^{\sim}\right)\right|=\left|m_{\alpha}\left(f * g^{\sim}-\varphi *\left(f * g^{\sim}\right)\right)\right| \\
& =\left|m_{\alpha}\left(f * g^{\sim}\right)-m_{\alpha}\left(l_{\psi}\left(f * g^{\sim}\right)\right)\right|
\end{aligned}
$$

(where $\left.\psi=(1 / \Delta) \varphi^{\sim} \in P(G)\right)=\left|\left(m_{\alpha}-l_{\psi}^{*} m_{\alpha}\right)\left(f * g^{\sim}\right)\right| \leqq\left\|m_{\alpha}-l_{\psi}^{*} m_{\alpha}\right\| \cdot\|f\|_{\infty}\|g\|_{1}$. Hence $\left\|\left(m_{\alpha}\right)_{R}(f-\varphi * f)\right\|_{\infty} \leqq\left\|m_{\alpha}-l_{\psi}^{*} m_{\alpha}\right\|\|f\|_{\infty} \rightarrow 0$. It follows that $\lim _{\alpha}\left\|\left(m_{\alpha}\right)_{R} f\right\|_{\infty}=0$ for any $f \in K$. If $f \in K^{-}$let $\varepsilon>0$, there is some $f_{0} \in K$ such that $\left\|f-f_{0}\right\|_{\infty}<\varepsilon$. Then

$$
\begin{aligned}
\left\|\left(m_{\alpha}\right)_{R} f\right\|_{\infty} & \leqq\left\|\left(m_{\alpha}\right)_{R}\left(f-f_{0}\right)\right\|_{\infty}+\left\|\left(m_{\alpha}\right)_{R} f_{0}\right\|_{\infty} \leqq\left\|f-f_{0}\right\|_{\infty}+\left\|\left(m_{\alpha}\right)_{R} f_{0}\right\|_{\infty} \\
& \leqq \varepsilon+\left\|\left(m_{\alpha}\right)_{R} f_{0}\right\|_{\infty} .
\end{aligned}
$$

Consequently $\lim _{\alpha}\left\|\left(m_{\alpha}\right)_{R} f\right\|_{\infty}=0$ for every $f \in K^{-}$.

Definition 7.2. Let $G$ be a locally compact group such that $L_{\infty}(G)$ has a topological left invariant mean. $f \in L_{\infty}(G)$ is said to be topological left almost convergent (t.l.a.c.) to $\beta$ iff $\mu(f)=\beta$ for every topological left invariant mean $\mu$ on $L_{\infty}(G)$.

In analogy to Granirer [10, Theorem III, 7] and following the idea there, one has

THEOREM 7.3. Let $G$ be a locally compact group for which $L_{\infty}(G)$ has a topological left invariant mean and $C$ the constants in $L_{\infty}(G)$. Then (a) $C \oplus \mathrm{K}^{-}$is the space of all topological left almost convergent functions in $L_{\infty}(G) . f$ being topological left almost convergent to $\beta$ iff $f \in \beta \cdot 1+K^{-}$. 
(b) If $f$ is topological left almost convergent to $\beta$ and $m_{\alpha}$ is any net of means on $L_{\infty}(G)$ such that $\lim _{\alpha}\left\|l_{\infty}^{*} m_{\alpha}-m_{\alpha}\right\|=0$ for any $\varphi \in P(G)$, then $\lim _{\alpha}\left\|\left(m_{\alpha}\right)_{R} f-\beta \cdot 1\right\|_{\infty}=0$.

(c) $f$ is topological left almost convergent to $\beta$ iff $\beta \cdot 1$ belongs to the norm closure of $P(G) * f$ in $L_{\infty}(G)$.

Proof. (a) Suppose $f$ is t.l.a.c. to zero, we claim that $f \in K^{-}$. Otherwise there would exist some $m \in L_{\infty}(G)^{*}$ such that $m\left(K^{-}\right)=0$ and $m(f) \neq 0$ by Hahn Banach Theorem. Hence $m$ is topological left invariant. Write $m=c_{1} m_{1}-c_{2} m_{2}$ where $m_{1}$, $m_{2}$ are means on $L_{\infty}(G)$ and let $\theta$ be any TLIM on $L_{\infty}(G)$ (which exists by assumption), we have $m=\theta \odot m=c_{1}\left(\theta \odot m_{1}\right)-c_{2}\left(\theta \odot m_{2}\right)$ by Lemma 4.1(g) where both $\theta \odot m_{1}$ and $\theta \odot m_{2}$ are TLIM on $L_{\infty}(G)$ by Lemma 4.1(f). Hence $m(f)=0$ which is a contradiction. Therefore $f \in K^{-}$.

On the other hand, if $m$ is any TLIM on $L_{\infty}(G)$, clearly $m$ annihilates $K^{-}$. Thus $K^{-}=\left\{f \in L_{\infty}(G): f\right.$ is t.l.a.c. to zero\}. Now $f$ is t.l.a.c. to $\beta$ iff $f-\beta \cdot 1$ is t.l.a.c. to zero, iff $f \in \beta \cdot 1+K^{-}$. To show that the sum $C+K^{-}$is $\operatorname{direct}$, let $\beta \cdot 1 \in K^{-}$ and choose any TLIM $m$ on $L_{\infty}(G)$. Then $\beta=m(\beta \cdot 1)=0$. This proves (a).

(b) Let $f$ be t.l.a.c. to $\beta$ and $m_{\alpha}$ a net of means on $L_{\infty}(G)$ such that

$$
\lim _{\alpha}\left\|l_{\Phi}^{*} m_{\alpha}-m_{\alpha}\right\|=0
$$

for any $\varphi \in P(G)$. Then $f-\beta \cdot 1 \in K^{-}$. By Lemma $7.1 \lim _{\alpha}\left\|\left(m_{\alpha}\right)_{R}(f-\beta \cdot 1)\right\|_{\infty}=0$. But $\left(m_{\alpha}\right)_{R}(\beta \cdot 1)=\beta \cdot 1$. Hence $\lim _{\alpha}\left\|\left(m_{\alpha}\right)_{R} f-\beta \cdot 1\right\|_{\infty}=0$.

(c) First we observe that if $f, g \in L_{\infty}(G)$ and $g \in P(G) * f$, then $m(f)=m(g)$ for any TLIM on $L_{\infty}(G)$. Therefore if $\beta \cdot 1$ belongs to the norm closure of $P(G) * f$, then $\beta=m(\beta \cdot 1)=m(f)$ for any TLIM $m$ on $L_{\infty}(G)$ or $f$ is t.l.a.c. to $\beta$. Conversely, if this is the case, let $\varphi_{\alpha}$ be a net in $P(G)$ such that $\lim _{\alpha}\left\|\varphi * \varphi_{\alpha}-\varphi_{\alpha}\right\|=0$ for any $\varphi \in P(G)$ [12, Theorems 2.4.2 and 2.4.3]. Since $\varphi * \varphi_{\alpha}=l_{\phi}^{*}\left(\varphi_{\alpha}\right)$ by Lemma 3.1(c), we have $\lim _{\alpha}\left\|l_{\varphi}^{*}\left(\varphi_{\alpha}\right)-\varphi_{\alpha}\right\|=0$ for any $\varphi \in P(G)$. But $f$ is t.l.a.c. to $\beta$, hence

$$
\lim _{\alpha}\left\|\left(\varphi_{\alpha}\right)_{R} f-\beta \cdot 1\right\|_{\infty}=0
$$

by (b). Now $\left(\varphi_{\alpha}\right)_{R} f=l_{\varphi_{\alpha}} f$. For if $g \in L_{1}(G),\left(\left(\varphi_{\alpha}\right)_{R} f\right) g=\varphi_{\alpha}\left(f * g^{\sim}\right)=\left(f * g^{\sim}, \varphi_{\alpha}\right)$ $=\left((1 / \Delta) \varphi_{\alpha} \tilde{\alpha} * f, g\right)$ or $\left(\varphi_{\alpha}\right)_{R} f=l_{\varphi_{\alpha}} f \in P(G) * f$. Consequently $\beta \cdot 1$ belongs to the norm closure of $P(G) * f$. This completes the proof of the theorem.

ReMARKS. (a) When $G$ is discrete, this is precisely Theorem III.7 of Granirer [10] restricted to groups.

(b) Let $W(G)$ be the space of all weakly almost periodic functions on $G$ (see $[12, \S 3.1]$ for definition). It is well known that $W(G)$ has a unique invariant mean which is also topological invariant, whether $L_{\infty}(G)$ has a TLIM or not. (For existence and uniqueness, consult Ryll-Nardzewski [22] and Deleeuw and Glicksberg [5].) Hence if $L_{\infty}(G)$ has a TLIM, $W(G) \subset C \oplus K^{-}$because every function in $W(G)$ is t.l.a.c. If $L_{\infty}(G)$ does not have a TLIM, then $K^{-}=L_{\infty}(G)$ and trivially $W(G) \subset C+K^{-}=L_{\infty}(G)$ although the sum is not direct. 
THEOREM 7.4. Let $G$ be any locally compact group such that $L_{\infty}(G)$ has a topological left invariant mean. Then $C \oplus K^{-}$is a topological left introverted and topological left invariant linear subspace of $L_{\infty}(G)$ containing the constants.

Proof. By definition $C \oplus K^{-}$contains the constants. It is topological left invariant because $C \oplus K^{-}$is precisely the topological left almost convergent functions in $L_{\infty}(G)$. To show that $C \oplus K^{-}$is topological left introverted, consider first functions of the form $f-\varphi * f$ with $f \in L_{\infty}(G), \varphi \in P(G)$. We have if $m \in L_{\infty}(G) *$, $m_{L}(f-\varphi * f)=m_{L}(f)-\varphi * m_{L}(f)=f_{1}-\varphi * f_{1}$ where $f_{1}=m_{L}(f) \in L_{\infty}(G)$ by Lemma 4.3(b). It follows that $m_{L}(K) \subset K$ and hence $m_{L}\left(K^{-}\right) \subset K^{-}$by continuity. Consequently $m_{L}\left(C \oplus K^{-}\right) \subset C \oplus K^{-}$.

Remarks. (a) The space $C \oplus K^{-}$contains functions which are not continuous. In fact the set $\left\{f-\varphi * f: f \in L_{\infty}(G)\right\}$ is not included in $C B(G)$ for any $\varphi$ provided that $L_{\infty}(G) \neq C B(G)$ (since $\varphi * f \in C B(G)$ ).

(b) If $L_{\infty}(G)$ has more than one topological left invariant mean. (This is true when $G$ is separable, noncompact and amenable as a discrete group. See [23, Theorem 1, p. 124 and Remark 1(a), p. 118].) Then not every function in $L_{\infty}(G)$ can be topological left almost convergent. Hence $C \oplus K^{-}$is a proper linear subspace of $L_{\infty}(G)$.

(c) Consider $G=R$ the additive reals with usual topology. Let

$$
E=\bigcup_{n=-\infty}^{\infty}[2 n, 2 n+1) \text { and } F=\bigcup_{n=-\infty}^{\infty}[2 n-1,2 n)
$$

Then $\chi_{E}, \chi_{F} \in L_{\infty}(G), \chi_{E}+\chi_{F}=1$ while $l_{1}\left(\chi_{E}\right)=\chi_{F}$ (i.e. $\chi_{F}$ is the left translate of $\chi_{E}$ by one). Hence both $\chi_{E}$ and $\chi_{F}$ are topological left almost convergent to $1 / 2$. In this case $C \oplus \mathrm{K}^{-}$even contains a characteristic function.

\section{REFERENCES}

1. R. F. Arens, The adjoint of a bilinear operation, Proc. Amer. Math. Soc. 2 (1951), 839-848.

2. M. M. Day, Amenable semigroups, Illinois J. Math. 1 (1957), 509-544.

3. - Fixed-point theorems for compact convex sets, Illinois J. Math. 5 (1961), 585-590.

4. - Correction to my paper "Fixed-point theorems for compact convex sets", Illinois J. Math. 8 (1964), 713.

5. K. Deleeuw and I. Glicksberg, Applications of almost periodic compactifications, Acta Math. 105 (1961), 63-97.

6. N. Dunford and J. T. Schwartz, Linear operators. Part I. General theory, Pure and Applied Math. No. 7, Interscience, New York, 1958.

7. W. F. Eberlein, Abstract ergodic theorems and weak almost periodic functions, Trans. Amer. Math. Soc. 67 (1949), 217-240.

8. E. Granirer, Extremely amenable semigroups, Math. Scand. 17 (1965), 177-197.

9. —_, Extremely amenable semigroups. II, Math. Scand. 20 (1967), 93-113.

10. - Functional analytic properties of extremely amenable semigroups, Trans. Amer. Math. Soc. 137 (1969), 53-76.

11. E. Granirer and Anthony Lau, A characterisation of locally compact amenable groups, Illinois J. Math. (to appear). 
12. F. P. Greenleaf, Invariant means on topological groups and their applications, Van Nostrand (to appear).

13. A. Grothendieck, Citeres de compacite dans les espaces functionnels genereaux, Amer. J. Math. 74 (1952), 168-186.

14. E. Hewitt and K. A. Ross, Abstract harmonic analysis. I: Structure of topological groups. Integration theory, group representations, Die Grundlehren der math. Wissenschaften, Bd. 115, Academic Press, New York and Springer-Verlag, Berlin, 1963.

15. A. Hulanicki, Means and Folner condition on locally compact groups, Studia Math. 27 (1966), 87-104.

16. J. Kelley, General topology, Van Nostrand, New York, 1955.

17. J. Kelley and I. Namioka et al., Linear topological spaces, Van Nostrand, New York, 1963.

18. T. Mitchell, Constant functions and left invariant means on semigroups, Trans. Amer. Math. Soc. 119 (1965), 244-261.

19. - Fixed points and multiplicative left invariant means, Trans. Amer. Math. Soc. 122 (1966), 195--202.

20. - Function algebras, means and fixed points, Trans. Amer. Math. Soc. 130 (1968), 117-126.

21. I. Namioka, On certain actions of semigroups on L-spaces, Studia Math. 29 (1967), 63-77.

22. C. Ryll-Nardzewski, On fixed points of semigroups of endomorphisms of linear spaces, Proceedings of the Fifth Berkeley Symposium on Math. Statistics and Probability. II, Berkeley, 1966.

23. E. E. Granirer, On the invariant mean on topological semigroups and on topological groups, Pacific J. Math. 15 (1965), 107-140.

24. R. Arens, Operations induced in function classes, Monatsh. Math. 55 (1951), 1-19.

25. P. Civin and B. Yood, The second conjugate space of a Banach algebra as an algebra, Pacific J. Math. 3 (1961), 847-870.

UNIVERSITY OF BRITISH COLUMBIA, Vancouver, B.C., Canada 